\title{
Power and Weakness of the Modal Display Calculus
}

\author{
Marcus Kracht
}

II. Mathematisches Institut

Freie Universität Berlin

Arnimallee 3

D-14195 Berlin

kracht@math.fu-berlin.de 


\section{Introduction}

The present paper explores applications of Display Logic as defined in [Belnap, 1982] to modal logic. Acquaintance with that paper is presupposed, although we will give all necessary definitions. Display Logic is a rather elegant proof-theoretic system that was developed to explore in depth the possibility of total Gentzenization of various propositional logics. By Gentzenization I understand the strategy to replace connectives by structures. Gentzenization is something of an ingenious optical trick because it uses a single symbol to mean different things depending on the place it occupies in the sequent. In the original Gentzen system it was the comma that had to be interpreted as and when to the left of the turnstile and as or when to the right. The interpretation of the structures oscillates between two logical symbols depending on whether it is in the antecedent or in the consequent. This is why we call symbols like comma Gentzen toggles. These two symbols between which this toggle switches are the Gentzen duals of each other. So, and and or are Gentzen duals. The strength of Display logic lies in a rather general cut-elimination theorem. In [Wansing, 1994b] and [Wansing, 1994a], Heinrich Wansing has refined these methods for modal logics; he showed that contrary to Belnap's own Gentzenization of modal operators as binary structure operators, a unary one is more appropriate (not only from an esthetical point of view) and makes perfect sense semantically as well. The Gentzen dual of the modal operator $\square$ is actually not - as one might expect - the possibility operator $\diamond$, but the backward looking possibility operator, denoted here by $\diamond$. (To be consistent with that we write $\square$ instead of $\square$ and $\diamond$ instead of $\diamond$.) The corresponding toggle is denoted by $\bullet$. The reason why this is so natural lies in the fact that it is the exact Display or Gentzen dual, for we have that the sequent $\bullet B \vdash A$ and the sequent $B \vdash \bullet A$ are equivalent if $\bullet$ is read as $\diamond$ if in the antecedent and if it is read as $\square$ if in the consequent. Wansing uses this fact to display various modal and tense logics à la Belnap by providing some formula introduction rules and basic structural rules for $\mathbf{K}$ and $\mathbf{K t}$ and then Gentzenizing the additional axioms. The benefit lies not only in the homogeneity with which all these systems are now handled and the rather clear intuitive background. The benefit lies in the possibility to use the general cut-elimination theorem of [Belnap, 1982].

During the summer of 1993 Rajeev Goré, Frank Wolter and myself have been intrigued by the possibility that Display Logic could be the key to rather simple decidability proofs via cut-elimination and some refined tricks of pushing around decidability. After successful proofs of the fact that all displayable logics are decidable - which we knew was wrong - and a subsequent investigation into the pos- 
sibility of having proved the inconsistency of arithmetic we found that one crucial lemma was flawed. The headache was soon to follow. Not only were the theorems on decidability false, it is actually undecidable whether a display calculus for modal logics is decidable.

The negative results are now assembled in [Kracht, 1995]. The present paper contains pretty much those parts of the original paper that have remained untouched by the disaster. I wish to thank Rajeev Goré for bringing my attention to Display Logic and his insistance that it is worth its while. Thanks for endlessly discussing this topic with me. Thanks also to Frank Wolter, Heinrich Wansing and Greg Restall or their criticism and to an anonymous referee for pointing out a number of mistakes in the first version.

\section{The Basic Calculus}

Below we outline the whole calculus for the basic tense logic Kt. This outline is a blend of [Belnap, 1982], [Wansing, 1994b] and some own ideas. Kt is a special bimodal logic in which we have two pairs of modal operators, namely $\square$ and $\diamond$, as well as $\square$ together with $\diamond$. The pairs look in opposite directions of the basic relation of the Kripke frame. We assume $\square$ and $\diamond$ to look with the relation denoted here by $\triangleleft$ and $\square$ and $\diamond$ to look in direction of its converse $\triangleright=\triangleleft$. The display calculus works with a special set of extra operators used to Gentzenize logical symbols into structures. These operators are $\mathbf{I}, \circ, *$ and $\bullet$. Given a set $\mathcal{L}$ of formulas we write $\operatorname{Struc}(\mathcal{L})$ for the algebra of structures over $\mathcal{L}$. $\operatorname{Struc}(\mathcal{L})$ is actually nothing but the term algebra over $\mathcal{L}$ with the operators $\mathbf{I}, *, \bullet$ and $\circ$. We distinguish formulas from structures from sequents. A sequent is of the form $X \vdash Y$ where both $X$ and $Y$ are structures. $X$ is called the antecedent and $Y$ the succedent. Succedents are called consequents in [Belnap, 1982] and instances of rules are called consecutions. Formulas are denoted by upper case letters such as $P, Q, R$ and structures by $X, Y, Z$. Sequents are pairs $X \vdash Y$ where $X$ and $Y$ are structures, and sequents are denoted by lower case letters such as $s, t$. We begin with the following fundamental logical axioms and rules

$$
\text { (Id) } p \vdash p \quad(\text { Cut }) \frac{X \vdash P \quad P \vdash Y}{X \vdash Y}
$$


and the following basic structural rules

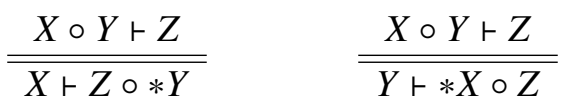

$$
\begin{aligned}
& \frac{X \vdash Y \circ Z}{X \circ * Z \vdash Y} \quad \frac{X \vdash Y \circ Z}{* Y \circ X \vdash Z} \\
& \frac{* X \vdash Y}{* * Y+X} \quad \frac{X \vdash * Y}{Y \vdash * X} \\
& \frac{* * X \vdash Y}{X \vdash Y} \quad \frac{X \vdash * * Y}{X \vdash Y} \\
& \frac{X \vdash \bullet Y}{\bullet-X \vdash Y}
\end{aligned}
$$

Notice that contrary to the established notation we write $*$ as a prefix, which makes formulae much easier to read. The following rules are now derivable.

$$
\frac{X \vdash Y}{* Y \vdash * X}
$$

These rules alone suffice to prove the following theorem, whose content gave rise to the name display logic.

Theorem 1 (Display Theorem) For every sequent $s$ and every antecedent (succedent) part $X$ of s there is a sequent $s^{\prime}$ structurally equivalent to $s$ such that $X$ is the antecedent (succedent) of $s^{\prime}$.

The correctness of this theorem depends on a proper definition of the terms antecedent part and succedent part. This is defined here via positive occurrence in, where an occurrence is positive if it is nested by an even number of $*$. Namely, if $P, Q$ are formulae then $* P \vdash * Q$ is a sequent but neither $P$ nor $Q$ can be displayed in their original position. However, we can easily derive $Q \vdash P$, displaying both $P$ and $Q$ on the other side. So if $s=V \vdash W$ is a sequent and $X$ occurred negatively in $V$, it will then occur positively in $* V$ and so it can be displayed in $* W \vdash * V$. The theorem is correct with the following definition as given in [Belnap, 1982].

Definition 2 In a sequent $V \vdash W$ an occurrence of $X$ is an antecedent part if it occurs positively in the antecedent or negatively in the succedent. An occurrence that is not an antecedent part is a succedent part. Equivalently, it is a succedent part if either it occurs positively in the succedent or negatively in the antecedent. 
It is actually possible to compute the sequent $s^{\prime}$ from $s$ and the occurrence of $X$. Namely, we can consider $s$ to be of the form $V \vdash W$, where $V$, say, contains an occurrence of $X$. This occurrence may be either positive or negative. Then we can think of $V$ as obtained from this occurrence of $X$ by applying only unary functions, for example prefixing with $\bullet$ or 'multiplying' from the left with $Y$, that is, applying the function $X \mapsto Y \circ X$, or multiplying with $Y$ from the right. Each of these functions has a dual function; the function $\bullet: X \mapsto \bullet X$ is it's own dual, $X \mapsto Y \circ X$ has the dual $X \mapsto * Y \circ X, X \mapsto X \circ Y$ has the dual $X \mapsto X \circ * Y$. * has no dual in this sense, which makes this story a bit complicated. Without $*$ we do the following. Let $V=f(X)$, where $f$ is a unary polynomial in the termalgebra of structures, and let $f^{\delta}$ be the dual of $f$. Then we transform $f(X) \vdash W$ into $X \vdash f^{\delta}(W)$. This transformation is reversible, so from $X \vdash f^{\delta}(W)$ we can also derive $f(X) \vdash W$. As noted, $*$ creates a problem. To undo $*$ we have to make antecedent and consequent swap sides, so we move from $* X \vdash W$ to $* W \vdash X$. With this proviso, it is justifiable to say that $*$ is self-dual. It is now clear why the Display Theorem has this peculiar restriction concerning the part-of relation, because for $X$ to be displayed on the same side as it occurred in the original sequent we need to perform this swapping an even number of times. The Display Theorem is brought here into the following form, writing $\mathbf{P o l}_{1}$ (Struc) for the set of unary structure polynomials.

Theorem 3 Let $f \in \boldsymbol{P o l}_{1}$ (Struc) which embeds an occurrence of its argument an even number of times with $*$. Then there exists a $f^{\delta} \in \boldsymbol{P o l}_{1}($ Struc) such that

$$
\frac{f(X) \vdash W}{X \vdash f^{\delta}(W)}
$$

displaying that particular occurrence of X. Moreover, for $f=g^{\delta}, f^{\delta}=g$ is an appropriate choice. $\dashv$

Let us note that the calculus has an inbuilt symmetry or self duality in the following sense. Define the dual $(X \vdash Y)^{\Delta}$ of a sequent by $(X \vdash Y)^{\Delta}=Y \vdash X$. The dual $\Pi^{\Delta}$ of a proof $\Pi$ consists in the dualization of all sequents.

Theorem 4 (Symmetry) For every proof $\Pi$ of $X \vdash Y, \Pi^{\Delta}$ is a proof of $Y \vdash X$.

Now we define the following equivalence between structures. $X$ and $Y$ are called similar, in symbols $X \approx Y$, if for every $Z$ the following two conditions hold.

$$
\frac{X \vdash Z}{\overline{Y \vdash Z}} \frac{Z \vdash X}{\overline{Z \vdash Y}}
$$


Similarity thus means that $X$ and $Y$ are interchangeable in a proof both as antecedent and succedent modulo some reversible rules. Replacement of $Y$ for $X$ in a given proof does not necessarily yield another proof but it can be made into one by adding some extra steps. The following theorem is a direct consequence of the Display Theorem.

Theorem $\mathbf{5} \approx$ is a congruence on $\operatorname{Struc}(\mathcal{L})$.

Proof. Clearly, $\approx$ is an equivalence relation. Moreover, as in our calculi we have full substitutivity, the only thing to be checked is whether $\approx$ satisfies the genuine congruence property that if $\sharp$ is an $n$-ary function symbol and $X_{1} \approx Y_{1}, \ldots, X_{n} \approx Y_{n}$ then also $\sharp\left(X_{1}, \ldots, X_{n}\right) \approx \sharp\left(Y_{1}, \ldots, Y_{n}\right)$. It can easily be seen that it is enough to show this for unary polynomials. Let $f$ be such a polynomial, and consider an occurrence of its argument $X$. Let $g$ be its dual with respect to this occurrence. Two cases have to be distinguished, namely whether or not $f(X)$ embeds $X$ an even number of times. Let the number be odd. Then we deduce from $f(X)+Z$ that $g(Z) \vdash X$ and then $g(Z) \vdash Y$, by assumption that $X \approx Y$, and then $f(Y) \vdash Z$. If $f$ embeds $X$ an even number of times, we deduce $X \vdash g(Z)$ instead, and then $Y \vdash g(Z)$ and finally $f(Y) \vdash Z$. Similarly for $X$ and $Y$ in the succedent. $\dashv$

\section{The Modal Display Calculus}

Let us now define the full calculus for the basic modal logic $\mathbf{K}$ and the basic tense logic Kt. It will be called DLM. In addition to the rules of the base calculus it has rules to introduce connectives and some more structural rules. The operational 
rules are the following

$$
\begin{aligned}
& (\vdash T) \mathbf{I} \vdash T \\
& (\vdash \perp) \frac{X \vdash \mathbf{I}}{X \vdash \perp} \\
& \text { ( 丁 }) \frac{\mathbf{I} \vdash X}{\top \vdash X}
\end{aligned}
$$

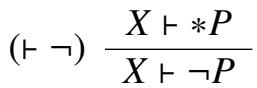

$$
\begin{aligned}
& (\perp \vdash) \perp \vdash \mathbf{I} \\
& (\vdash \wedge) \frac{X \vdash P \quad Y \vdash Q}{X \circ Y \vdash P \wedge Q} \\
& (\neg \vdash) \frac{* P \vdash X}{\neg P \vdash X} \\
& (\vdash \vee) \frac{X \vdash P \circ Q}{X \vdash P \vee Q} \\
& (\wedge \vdash) \frac{P \circ Q \vdash X}{P \wedge Q \vdash X} \\
& (\vdash \rightarrow) \frac{X \circ P \vdash Q}{X \vdash P \rightarrow Q} \\
& (\vee \vdash) \frac{P \vdash X \quad Q \vdash Y}{P \vee Q \vdash X \circ Y} \\
& \text { (トロ) } \frac{\bullet X \vdash P}{X \vdash \square P} \\
& (\rightarrow \vdash) \frac{X \vdash P \quad Q \vdash Y}{P \rightarrow Q \vdash * X \circ Y} \\
& (\vdash \diamond) \frac{X \vdash P}{* \bullet * X \vdash \diamond P} \\
& \text { (๘ト) } \frac{P \vdash X}{\square P \vdash \bullet X} \\
& (\diamond \vdash) \frac{P \vdash \bullet X}{\diamond P \vdash X} \\
& (\diamond \vdash) \frac{* \bullet * P \vdash X}{\diamond P \vdash X} \\
& \text { (घト) } \frac{P \vdash X}{\square P \vdash * \bullet * X} \\
& (\vdash \diamond) \frac{X \vdash P}{\bullet X \vdash \diamond P} \\
& (\vdash \boxminus) \frac{X \vdash * \bullet * P}{X \vdash \boxminus P}
\end{aligned}
$$

The calculus for $\mathbf{K}$ will be obtained by deleting the introduction rules for the connectives $\square$ and $\diamond$. Notice the complete duality between $\square$ and $\diamond$ as well as between $\diamond$ and $\square$. We can formalize it by extending the duality map as follows

$$
\begin{aligned}
& p^{\Delta} \quad=p \quad(\neg P)^{\Delta} \quad=\neg\left(P^{\Delta}\right) \\
& (P \wedge Q)^{\Delta}=P^{\Delta} \vee Q^{\Delta} \quad(P \vee Q)^{\Delta}=P^{\Delta} \wedge Q^{\Delta} \\
& (\square P)^{\Delta} \quad=\diamond\left(P^{\Delta}\right) \quad(\diamond P)^{\Delta} \quad=\quad{ }_{\square}\left(P^{\Delta}\right) \\
& (\boxminus P)^{\Delta} \quad=\diamond\left(P^{\Delta}\right) \quad(\diamond P)^{\Delta} \quad=\quad \square\left(P^{\Delta}\right) \\
& \mathbf{I}^{\Delta} \quad=\mathbf{I} \quad(* X)^{\Delta}=*\left(X^{\Delta}\right) \\
& (X \circ Y)^{\Delta}=X^{\Delta} \circ Y^{\Delta} \quad(\bullet X)^{\Delta} \quad=\bullet\left(X^{\Delta}\right)
\end{aligned}
$$

Theorem 6 (Duality) For every proof $\Pi$ of $X \vdash Y, \Pi^{\Delta}$ is a proof of $Y^{\Delta} \vdash X^{\Delta} . \dashv$

Of course, this time we can only speak of duality, not of symmetry. Finally, the following structural rules are added. (If only a part of these rules is added, we have a substructural calculus. Much of what will be proved here applies to substructural 
logics as well; in a sense, the full calculus is the most difficult case, and this is the reason why we concentrate on this calculus.)
(I $l) \frac{X \vdash Z}{\mathbf{I} \circ X \vdash Z}$
(Ir) $\frac{X \vdash Z}{X \vdash \mathbf{I} \circ Z}$
$(Q l) \frac{\mathbf{I} \vdash Y}{* \mathbf{I} \vdash Y}$
$(Q r) \frac{X \vdash \mathbf{I}}{X \vdash * \mathbf{I}}$
(Wl) $\frac{X \vdash Z}{Y \circ X \vdash Z}$
$(W r) \frac{X \vdash Z}{X \circ Y \vdash Z}$
(Al) $\frac{X_{1} \circ\left(X_{2} \circ X_{3}\right) \vdash Z}{\left(X_{1} \circ X_{2}\right) \circ X_{3} \vdash Z}$
(Ar) $\frac{Z \vdash X_{1} \circ\left(X_{2} \circ X_{3}\right)}{Z \vdash\left(X_{1} \circ X_{2}\right) \circ X_{3}}$
(Pl) $\frac{X \circ Y \vdash Z}{Y \circ X \vdash Z}$
(Pr) $\frac{Z \vdash X \circ Y}{Z \vdash Y \circ X}$
(Cl) $\frac{X \circ X \vdash Z}{X \vdash Z}$
(Cr) $\frac{Z \vdash X \circ X}{Z \vdash X}$
$(M l) \frac{\mathbf{I} \vdash Y}{\bullet \mathbf{I} \vdash Y}$
$(M r) \frac{X \vdash \mathbf{I}}{X \vdash \bullet \mathbf{I}}$

This concludes the definition of DLM. There are a number of things which have to be explained. First of all, some rules originally proposed in [Wansing, 1994b] have been left out because they are derivable. On the other hand, a lot of structural rules have been added to the calculus, mainly the duals of existing rules. The onesided rules are sufficient, but this has the effect of disturbing the duality on the level of proofs (not for provability, since the two-sided rules are derivable from the one-sided rules). The necessitation rules have been changed; they are now fully structural and do not require the use of formula variables. Furthermore, there are now two more necessitation rules derivable with the help of the newly introduced rules $(Q)$, namely

$$
(D l) \frac{\mathbf{I} \vdash Y}{* \bullet * \mathbf{I} \vdash Y} \quad(D r) \frac{X \vdash \mathbf{I}}{X \vdash * \bullet * \mathbf{I}}
$$


For example, the first is derived as follows

$$
\begin{gathered}
X{ }^{X} \mathbf{I} \\
\hline X \vdash * * \mathbf{I} \\
\hline * \mathbf{I} \vdash * X \\
\hline \mathbf{I} \vdash * X \\
\hline \bullet \mathbf{I} \vdash * X \\
\hline \mathbf{I} \vdash \bullet * X \\
\hline * \mathbf{I} \vdash \bullet * X \\
\hline \bullet * \mathbf{I} \vdash * X \\
\hline X \vdash * \bullet * \mathbf{I}
\end{gathered}
$$

Define the following translation for sequents.

$$
\begin{array}{llll}
\multicolumn{5}{c}{\tau(X \vdash Y)=\tau_{1}(X) \rightarrow \tau_{2}(Y)} & \\
\tau_{1}(P) & =P & \tau_{2}(P) & =P \\
\tau_{1}(\mathbf{I}) & =\top & \tau_{2}(\mathbf{I}) & =\perp \\
\tau_{1}(* X) & =\neg \tau_{2}(X) & \tau_{2}(* X) & =\neg \tau_{1}(X) \\
\tau_{1}(X \circ Y) & =\tau_{1}(X) \wedge \tau_{1}(Y) & \tau_{2}(X \circ Y) & =\tau_{2}(X) \vee \tau_{2}(Y) \\
\tau_{1}(\bullet X) & =\diamond \tau_{1}(X) & \tau_{2}(\bullet X) & =\square \tau_{2}(X)
\end{array}
$$

With respect to this translation we have the following theorem.

Lemma 7 Let $X \vdash Y$ be a sequent. From $X \vdash Y$ the sequent $I \vdash \tau_{1}(X) \rightarrow \tau_{2}(Y)$ is derivable in $D L M$.

Proof. We prove that if $X \vdash Y$ is derivable, then so is $\tau_{1}(X) \vdash \tau_{2}(Y)$. It is not to put the proof to a conclusion from there. The remaining proof is an induction on the complexity of the sequent. In fact, we will again prove something stronger, namely that we can derive any $V \vdash W$ which arises from $X \vdash Y$ by replacing any given substructure by its proper translation (i. e. by its $\tau_{1}$-translation or by its $\tau_{2}$ translation depending on whether it occurs as antecedent part or as succedent part). We will not go through all the cases. But take the case of a sequent $X \circ Y \vdash Z$ and assume that the claim has been verified for $X$ and $Y$. We wish to show it for $X \circ Y$. 
Here is a proof.

\begin{tabular}{c}
$\frac{X \circ Y \vdash Z}{X \vdash Z \circ * Y}$ \\
\hline$\frac{\tau_{1}(X) \vdash Z \circ * Y}{Y Y \vdash * \tau_{1}(X) \circ Z}$ \\
\hline$\frac{\tau_{1}(Y) \vdash * \tau_{1}(X) \circ Z}{\tau_{1}(X) \circ \tau_{1}(Y) \vdash Z}$ \\
\hline$\frac{\tau_{1}(X) \wedge \tau_{2}(Y) \vdash Z}{\tau_{1}(X \circ Y) \vdash Z}$
\end{tabular}

Another interesting case is $\bullet X \vdash Z$.

$$
\begin{gathered}
\frac{\bullet X \vdash Z}{X+\bullet Z} \\
\hline \tau_{1}(X) \vdash \bullet Z \\
\hline \diamond \tau_{1}(X) \vdash Z \\
\hline \tau_{1}(\bullet X) \vdash Z
\end{gathered}
$$

It is easy to supply the remaining cases. -1

Theorem 8 A sequent $X \vdash Y$ is DLM-provable iff $\tau(X \vdash Y)$ is a theorem of the tense logic $\mathbf{K t}$.

Proof. The correctness of the display calculus is a matter of straightforward verification. Notice that the display calculus contains modulo translation only rules which are derived rules of $\mathbf{K t}$, such as $\left(M N^{+}\right) P / \square P$, and $\left(M N^{-}\right) P / \square P$, which are consequences of the rules $(\mathrm{Ml})$ and $(\mathrm{Mr})$ (modulo some other rules). The completeness is somewhat tricky. Consider the set $\Theta$ of all formulae $P$ such that $\mathbf{I} \vdash P$ is derivable in DLM. Moreover, consider the set $\Theta_{\vdash}$ of all $\tau(X \vdash Y)$ which are derivable in DMLE. By Lemma 9 below we know that a sequent is derivable in DLME iff it is derivable in DLM, and that sequents with identical $\tau$-translation are interderivable. We conclude that $\Theta=\Theta_{\vdash}$. Now it remains to be seen that $\Theta$ is a normal modal logic, which will establish the completeness. If $P \in \Theta$, then also ${ }_{\square} P \in \Theta$ and $\square P \in \Theta$, as can easily be shown. Next, if $P \in \Theta$ and $P \rightarrow Q \in \Theta$, then not only $\mathbf{I} \vdash P \rightarrow Q$ is derivable, but also $P \vdash Q$. By an application of (Cut) we get I $\vdash Q$, showing $Q \in \Theta$. It still needs to be shown that all boolean tautologies can be proved, but we refer here to [Wansing, 1994b]. $\dashv$ Here are the additional rules 
for the calculus DLME.

$$
\begin{array}{rlrl}
(\text { (ト) } & \frac{P \wedge Q \vdash Y}{P \circ Q \vdash Y} & (\vdash \circ) & \frac{X \vdash P \vee Q}{X \vdash P \circ Q} \\
(* \vdash) & \frac{\neg P \vdash Y}{* P \vdash Y} & (\vdash *) & \frac{Y \vdash \neg P}{Y \vdash * P} \\
(\mathbf{I} \vdash) & \frac{T \vdash Y}{\mathbf{I} \vdash Y} & (\vdash \mathbf{I}) \frac{X \vdash \perp}{X \vdash \mathbf{I}} \\
(\bullet \vdash) & \frac{\diamond P \vdash Y}{\bullet P \vdash Y} & (\vdash \bullet) & \frac{X \vdash \square P}{X \vdash \bullet P} \\
(* \bullet *) & \frac{\diamond P \vdash Y}{* \bullet * P \vdash Y} & (\vdash * \bullet *) & \frac{X \vdash \square Q}{X \vdash * \bullet * Q}
\end{array}
$$

Lemma 9 All rules of $D L M E$ are admissible in $D L M$. Moreover, if $X_{1} \vdash Y_{1}$ and $X_{2} \vdash Y_{2}$ have identical $\tau$-translation, then they are interderivable in $\mathbf{D L M E}$.

Proof. The proof is by showing that the highest application in a proof tree of such a rule can be eliminated without adding new instances of such rules. To make life simple, we will assume that DLM admits cut-elimination. (This will be proved in the next section. This assumption is not strictly speaking necessary, but it simplifies the argument.) Thus we can assume our proofs to contain no cuts. Now take a highest instance of an elimination rule, say of ( $\circ \vdash)$. It's premiss is of the form $P \wedge Q \vdash Y$. Now trace the occurrences of $P \wedge Q$ backwards. Each occurrence below the line has one (in the case of contraction two) counterparts above the line, unless, of course, $P \wedge Q$ is principal (see next section for a definition). This can only be in an application of $(\wedge \vdash)$. Replacing the traced occurrences of $P \wedge Q$ by $P \circ Q$ will transform valid instances of rules in valid ones, with the exception when $P \wedge Q$ is principal. Then replacing $P \wedge Q$ below by $P \circ Q$ will result in a trivial deduction, which can be omitted. $\dashv$

\section{Cut-Elimination and the Subformula Property}

[Belnap, 1982] lists eight conditions on a proper display logic called $(C 1)-(C 8)$ and proves the following.

Theorem 10 Any display calculus satisfying (C1) has the subformula property, that is, any cut-free proof of the sequent $X \vdash Y$ contains only structures over subformulas of formulas in $X$ and $Y$. 
Theorem 11 In any display calculus satisfying $(C 2)-(C 8)(C u t)$ is eliminable.

Now what are these conditions $(C 1)-(C 8)$ ? We will give the original conditions in some slightly less general form. The difference is that we have stated a rule of simultaneous substitution $(C 6 / 7)$, which is the appropriate to choice to make in this context. Belnap assumes that in each rule we first stipulate a set of constituents and an equivalence relation on parameters called congruence. Here, parameter is an occurrence of a structure in a rule.

(C1) Each formula which is a constituent of some premiss of a rule $\rho$ is a subformula of some formula in the conclusion of $\rho$.

(C2) Congruent parameters are occurrences of the same structure.

(C3) Each parameter is congruent to at most one constituent in the conclusion. Equivalently, no two constituents of the conclusion are congruent to each other.

(C4) Congruent parameters are either all antecedent or all succedent parts of their respective sequent.

(C5) If a formula is nonparametric in the conclusion of a rule $\rho$ it is either the entire antecedent or the entire succedent. Such a formula is called principal formula of $\rho$.

(C6/7) Each rule is closed under simultaneous substitution of arbitrary structures for congruent parameters.

(C8) If there are inference rules $\rho$ and $\sigma$ with respective conclusions $X \vdash P$ and $P \vdash Y$ with $P$ principal in both inferences (in the sense of $C 5$ ) and if (Cut) is applied to yield $X \vdash Y$ then either $X \vdash Y$ is identical to either $X \vdash P$ or $P \vdash Y$; or it is possible to pass from the premisses of $\rho$ and $\sigma$ to $X \vdash Y$ by means of inferences falling under $(\mathrm{Cut})$ where the cut-formula always is a proper subformula of $P$.

These conditions actually need some exegesis. First of all, the present formulation assumes that the so-called analysis is performed at the actual proofs, not at the rules as presented above. To see the difference, let us call a rule a consecution, and a rule skeleton a consecution formulated with the help of structure variables. A rule skeleton can be instantiated to a rule by substituting structures for structure vaiables. Notice that rules still contain variables, but only for formulae. We 
assume that any substitution instance of structures for structure variables and of formulas for formula variables is an instance of the rule. Rather than applying the analysis to the instances of the rules (which have no structure variables!), we perform the anaysis on the rule skeleta of the calculus DLM as presented above. Hence, for our purposes, a parameter may be thought of as an occurrence of a structure variable in a rule skeleton. In the actual Display Calculus, however, they are merely metavariables standing in for arbitary occurrences of structures. This is the way we will handle the Display Logic later as well. But let us suppose for the moment that they are genuine variables of the system. Then formula variables can never be parameters. Furthermore, if a structure $X$ occurs parametric once, then all occurrences of $X$ are parametric. Under our interpretation, then, $(C 2)$ is trivially satisfied. Moreover, $(C 6 / 7)$ is satisfied as well by force of our definition. Only the remaining conditions are ever to be checked in DLM. In the associativity law $(A)$, for example, all variables are parameters, and all occurrences of the same variable are congruent. In a formula introduction rules such as $(\vdash \wedge)$ the new formula in the conclusion as well as the old ones in the premiss are typically not parameters.

With the exception of $(C 8)$ the conditions are verified by direct inspection. We leave it to the reader to verify $(C 1),(C 3),(C 4)$ and $(C 5)$. For $(C 8)$, [Wansing, 1994b] gives a proof that $(C 8)$ holds for $\diamond Q$ and $\square Q$ as cut-formulas. The case of $\square Q$ and $\diamond Q$ is completely dual, i. e., obtained by swapping antecedent with succedent, so we might actually skip the proof here, but for the sake of completeness we give the corresponding proof of $(C 8)$ in these cases. So let us first suppose that the cut-formula is $\diamond Q$. Then we have

$$
\frac{\frac{X \vdash Q}{\bullet X \vdash \diamond Q} \frac{Q \vdash \bullet Y}{\diamond Q \vdash Y}}{\bullet X \vdash Y}
$$

The following, however, is a proof involving a cut on $Q$ rather than $\diamond Q$.

$$
\frac{X \vdash Q \quad Q \vdash \bullet Y}{\frac{X \vdash \bullet Y}{\bullet X \vdash Y}}
$$

Suppose next that we have a proof involving $\square Q$ as a cut-formula.

$$
\frac{\frac{X \vdash * \bullet * Q}{X \vdash \boxminus Q} \frac{Q \vdash Y}{\square Q \vdash * \bullet * Y}}{X \vdash * \bullet * Y}
$$


The following is a proof with a cut on $Q$.

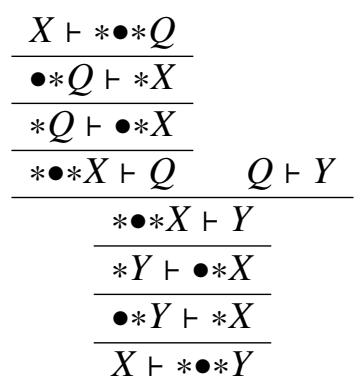

\section{Properly Displaying Extensions of Kt}

The usefulness of Display Logic shall be demonstrated with a theorem which shows that a large class of logics have a canonical proof system in Display Logic. We will analyse exactly which rules $\delta=P_{1} \ldots P_{m} / Q$ can be incorporated into DLM by just adding another structural rule that does not destroy the properties $(C 1)-(C 8)$. Such a rule captures a rule via its $\tau$-translation in modal logic as follows. Let us be given a $\operatorname{logic} \Lambda=\mathbf{K t}+\Delta$ that is, an extension of (Hilbert-style) tense logic by a set of rules $\Delta$, and assume that we add to DLM a set $R$ of structural rules. Then we say that $\mathbf{D L M}+R$ properly displays $\mathbf{K t}+\Delta$ if $(C 1)-(C 8)$ are satisfied and every derived rule of $\mathbf{K t}+\Delta$ is the $\tau$-translation of a derived rule of $\mathbf{D L M}+R$. The latter condition can be rephrased as requiring that a rule transition is derivable in DLME $+R$ iff its $\tau$-translation is a derived rule of $\mathbf{K t}+\Delta$. For, by the fact that the added structural rules preserve the properties $(C 1)-(C 8)$ we have cutelimination, and an analogue of Lemma 9 holds. Then in DLME $+R, \tau$-equivalent sequents are interderivable, while in DLM $+R$ we can only go from sequents to sequents if we do not eliminate connectives. Nevertheless, it is easy to see that if a rule is DLME $+R$-derivable, then there is another rule with identical $\tau$-translation which is derivable in DLM $+R$. In this section we will give a complete characterization of properly displayable Kt-calculi. We show first that the contribution of a rule $\rho=s_{1} s_{2} \ldots s_{m} / t$ can be directly computed as $\check{\rho}=\tau\left(s_{1}\right) \ldots \tau\left(s_{m}\right) / \tau(t)$.

Lemma 12 Suppose that $\rho$ is a structural rule, and let $D L M+\rho$ satisfy $(C 1)-$ (C8). Then $\mathbf{D L M}+\rho$ properly displays $\boldsymbol{K} \boldsymbol{t}+\check{\rho}$.

Proof. Two things need to be seen. First, any extension by structural rules satisfying the display conditions axiomatizes a normal logic. And second, that it 
axiomatizes the logic as given. Let $\Theta$ be the set of all $\tau(X \vdash Y)$ which are derivable in DLME $+\rho$. Since the rules of the calculus turn into axioms of $\mathbf{K t}+\check{\rho}$, we have proved now that we have displayed a logic at most as strong as $\mathbf{K t}+\check{\rho}$. On the other hand, suppose that for some substitution $\sigma$ we have proved $P_{1}^{\sigma}, \ldots, P_{m}^{\sigma}$. then there is a substitution $\omega$ of formulae to structure variables such that $P_{i}^{\sigma}=\tau\left(s_{i}{ }^{\omega}\right)$. Then if we can derive $\tau\left(s_{i}{ }^{\omega}\right)$ we can also derive $\tau\left(t^{\omega}\right)=Q^{\sigma}$. Thus $\check{\rho}$ is a derived rule of the calculus DLM $+\rho$ translated under $\tau$. $\dashv$

For example, adding the rule

$$
\frac{\mathbf{I} \vdash \bullet X}{\text { I } \vdash X}
$$

This rule translates into the rule $\square P / P$, a rule which is actually admissible in $\mathbf{K t}$, but not derivable. In the calculus obtained by $\tau$-translation it will however be a derived rule.

Now, modal logics are generally studied as axiomatic strengthenings of a basic system rather than a strengthening by proper rules. Therefore, let us concentrate on the question of displayability of axiomatic extensions. First we will consider which axioms admit a resolution into a structural rule. Denote by $\mathbf{K t} \oplus \delta$ the least normal logic extending Kt which also contains $\delta$. $\delta$ can always be written in the form $A \rightarrow B$, where $A$ and $B$ are free of $\rightarrow$. We can then pass from the axiom $A \rightarrow B$ to a sequent rule

$$
\frac{B \vdash Y}{A \vdash Y}
$$

This sequent rule is as powerful as the axiom. For putting $Y=p$, a variable not contained in $A$ or $B$ the $\tau$-translation is the rule $\tau_{1}(B) \rightarrow p / \tau_{1}(A) \rightarrow p$. Now let $p=\tau_{1}(B)$; then the premiss of $\breve{\rho}$ becomes a theorem and we see that $\tau_{1}(A) \rightarrow \tau_{1}(B)$ is an axiom of the calculus axiomatized by this rule. The axiom allows to derive the rule, however, and so the two are equal in power. Assume now that both $A$ and $B$ are composed from $T$ and propositional variables using only $\wedge, \vee, \diamond$ and $\diamond$. Then, by standard equivalences,

$$
A . \leftrightarrow . \bigvee_{i \leq m} C_{i} \quad B . \leftrightarrow . \bigvee_{j \leq n} D_{j}
$$

where all $C_{i}, D_{j}$ are composed from variables and $\top$ with the help of $\wedge, \diamond$ and $\diamond$ only. (Thus, disjunction has been eliminated.) Instead of the rule above we then equip our calculus with the rules $\rho_{1}, \ldots, \rho_{m}$ where each $\rho_{i}$ is of the form

$$
\frac{D_{1} \vdash Y, \ldots, D_{n} \vdash Y}{C_{i} \vdash Y}
$$


Again it is checked that this new calculus is equivalent in power with our logic. Finally, define the translation $\sigma$ from formulae into structures via

$$
\begin{array}{ll}
\sigma(T) & =\mathbf{I} \\
\sigma(p) & =p \\
\sigma(P \wedge Q) & =\sigma(P) \circ \sigma(Q) \\
\sigma(\diamond P) & =\bullet \sigma(P) \\
\sigma(\diamond P) & =* \bullet * \sigma(P)
\end{array}
$$

It is checked by induction that $\tau_{1}(\sigma(P))=P$ for every formula $P$ made from variables, $T$, conjunction and possibility operators. Thus we have eliminated $\vee$. Finally, then, let us replace the above rules $\rho_{i}$ by their Gentzenized sisters.

$$
\frac{\sigma\left(D_{1}\right) \vdash Y, \ldots, \sigma\left(D_{n}\right) \vdash Y}{\sigma\left(C_{i}\right) \vdash Y}
$$

So, for axioms of the form $A \rightarrow B$, where $A$ and $B$ are positive and free of $\square$ and $\square$ we have managed to write a display system that completely axiomatizes it. For the rules above $(C 1)$ is obviously satisfied since we are strengthening the system for $\mathbf{K t}$ by structural rules. $(C 4)$ and $(C 5)$ are verified by the eye. The condition $(C 8)$ has to be checked only with respect to the rules which are introducing formulae, and this has been done already. We are left with $(C 3)$. (C3) is actually not automatically valid. In fact, we must place the restriction on the formula $A$ that it may contain each variable only once. (Maybe only those which already occur in $B$, but the others can be eliminated.) Let us now agree to call a formula primitive if it is of the form $A \rightarrow B$ where both $A$ and $B$ contain only variables, $\mathrm{\top}, \wedge, \vee, \diamond$ and $\diamond$ and that $A$ contains each propositional variable at most once.

Lemma 13 Suppose $\Lambda$ is a tense logic axiomatizable by primitive axioms. Then $\Lambda$ can be properly displayed.

Proof. $\Lambda$ can be displayed in the way described above; the display calculus meets $(C 1)-(C 8)$ and therefore enjoys cut-elimination and the subformula property. The system is complete for $\Lambda$ in the sense that it derives $X \vdash Y$ iff $\tau_{1}(X) \vdash_{\Lambda} \tau_{2}(Y)$.

Now let us tackle the question what logics are defined by a display calculus. We will show that it is actually the same class of logics if we insist that additional rules are completely structural. To see this consider a rule

$$
\rho \quad \frac{X_{1} \vdash Y_{1}, \ldots, X_{n} \vdash Y_{n}}{V \vdash W}
$$


where all $X_{i}, Y_{j}, W$ contain only structure variables. By $(C 4)$, congruent formulae do not swap sides, and so they are either in the antecedent throughout or in the conclusion throughout. Now rewrite this rule into the following form.

$$
\rho^{*} \quad \frac{\mathbf{I} \vdash * X_{1} \circ Y_{1}, \ldots, \mathbf{I} \vdash * X_{n} \circ Y_{n}}{\mathbf{I} \vdash * V \circ W}
$$

$\rho$ and $\rho^{*}$ are equivalent and $\rho^{*}$ satisfies $(C 4)$ as well as $(C 3)$ if $\rho$ does. The other conditions are harmless. This rule translates into the axiom

$$
\tau_{2}\left(* X_{1} \circ Y_{1}\right), \ldots, \tau_{2}\left(* X_{n} \circ Y_{n}\right) / \tau_{2}(* V \circ W)
$$

so that we now have to worry about which formula can occur as a translation of a structure under $\tau_{2}$. Dually, we can solve the question of which formulae are translations under $\tau_{1}$.

Lemma $14 P=\tau_{1}(X)$ for some $X$ containing no formula connectives exactly if $P \dashv Q$ for some $Q$ built from variables and negated variables, $\mathrm{T}, \wedge$ and the diamonds $\diamond$ and $\diamond$.

Proof. We know that if $X \approx Y$ then $\tau_{1}(X) \dashv \vdash \tau_{1}(Y)$ so that without loss of generality we can assume that $X$ is in normal form (see Section 9)

$$
X=p_{i_{1}} \circ \ldots \circ p_{i_{k}} \circ * p_{j_{i}} \circ \ldots \circ * p_{j_{l}} \circ \bullet X_{i} \circ \ldots \circ \bullet X_{m} \circ * \bullet Y_{1} \circ \ldots \circ * \bullet Y_{n}
$$

Then

$$
\tau_{1}(X)=\bigwedge_{r \leq k} p_{i_{r}} \wedge \bigwedge_{s \leq l} \neg p_{j_{s}} \wedge \bigwedge_{t \leq m} \diamond \tau_{1}\left(X_{t}\right) \wedge \bigwedge_{u \leq n} \diamond \tau_{1}\left(* Y_{u}\right)
$$

Now do induction on the number of nested occurrences of $\bullet$. $\dashv$

There remains now only the problem of negated occurrences of variables to prove the characterization. Therefore consider a variable $p$ that occurs negated in $\tau_{1}(V \circ$ $* W$ ). Then replace $p$ in the axiom throughout by $\neg p$. As it occurs negated throughout (by (C4) and the definition of part of), after substitution it occurs doubly negated throughout and so we can eliminate the negation altogether for $p$. Similarly for variables occurring only in the premisses. Thus we have proved the following characterization.

Theorem 15 An extension of $\boldsymbol{K t}$ by rules can be properly displayed iff each rule $P_{1} \ldots P_{m} \vdash Q$ is such that all occurring formulae are built from $\perp$ and variables with the help of $\wedge, \vee$ and $\square, \square . \dashv$ 
We can obtain as a special corollary a characterization of properly displayable axiomatic extensions. By the condition of completeness of DLME $+\check{\rho}$ with respect to the logic $\mathbf{K t} \oplus \delta$ it is possible to display $\mathbf{K t} \oplus \delta$ by rules of the form

$$
\rho \quad \frac{X_{1} \vdash Y, \ldots, X_{n} \vdash Y}{V \vdash Y}
$$

Call these rules special. They are characterized by the fact that one variable is shared as the common succedent or antecedent. For on the one hand, putting $Y=p$, a fresh variable, the rule corresponding to $\rho$ is

$$
\check{\rho} \quad \tau_{1}\left(X_{1}\right) \rightarrow p, \ldots, \tau_{1}\left(X_{n}\right) \rightarrow p / \tau_{1}(V) \rightarrow p
$$

Putting $p=\bigvee_{i} \tau_{1}\left(X_{i}\right)$ we derive the axiom $\tau_{1}(V) \rightarrow \bigvee_{i} \tau_{1}\left(X_{i}\right)$. This is a primitive formula. This formula if taken as an axiom is as least as strong as the rule $\check{\rho}$. So, by the completeness of DLM $+\rho$ for $\mathbf{K t}+\check{\rho}$ we get the completeness for $\mathbf{K t} \oplus \tau_{1}(V) \rightarrow \bigvee_{i} \tau_{1}\left(X_{i}\right)$. On the other hand, any axiom can be brought into the form $A \rightarrow B$, and hence is characterized by a special rule. This special rule must be derivable in DLME. By the condition that $A$ and $B$ are translations of structures, we ultimately arrive as before at the requirement that $A \rightarrow B$ is primitive. But then it can be properly displayed, as we have seen.

Theorem 16 (Proper Display I) An axiomatic extension of Hilbert-style tense logic can be properly displayed (by structural rules over DLM) iff it is axiomatizable by a set of primitive axioms. $\dashv$

\section{Semantic Characterization}

It is possible to characterize exactly the semantic conditions that can be associated with primitive axioms. It is known from correspondence theory ([Sahlqvist, 1975] and [Benthem, 1984]) that primitive formulae are canonical and therefore complete; in addition the condition that the formula places on the canonical and Kripke frames is elementary. Yet it is also important to know what condition a particular axiom expresses. [Benthem, 1984] developed the method of substitutions to find the elementary equivalent of a Sahlqvist formula but we find this method not so user-friendly. Instead we use the technique of decisive sets as proposed in [Kracht, 1993]. Some results have also been established in the somewhat simpler [Kracht, 1991]. This method rests on the fact that the condition expressed by an axiom can be squeezed out by very special valuations. These valuations decide what elementary condition this axiom determines in the sense that to know what the condition 
is we just have to check these valuations. Equivalently, to test a Kripke frame for whether it accepts a given Sahlqvist formula we only have to use these decisive sets as values of the propositions (or, even better, this can be fine tuned to decisive valuations rather than decisive sets). On Kripke frames the question is then settled. On generalized frames we have two choices: either these valuations exist - and then the elementary condition is forced on the underlying Kripke frame by these general arguments - or the sets are the infinite intersection of admissible valuations. In this case if the frame is compact as a topological space it guarantees that a family of valuations converging to a decisive valuation has the same effect as the decisive valuation itself. In this case we can simply pretend that the decisive sets are internal in the generalized frame. (Or, equivalently, that they can be added without disturbing the sets of Sahlqvist formulae accepted by the frame.)

It turns out that for the axioms in question the singleton sets $\{w\}, w$ a world, are decisive. This is most welcome in practical computation, because then we can do what every student of modal logic (including the author) is at one stage always tempted to do; namely, to pretend that a variable stands for being at a certain world. For example, in the axiom $\diamond p \wedge \diamond q . \rightarrow . \diamond(p \wedge q)$ let us pretend $p$ means being at $x_{p}$ and $q$ means being at $x_{q}$. Then this axiom tells us that if we can see $x_{p}$ and $x_{q}$ then $x_{p}$ and $x_{q}$ must be equal. Hence, it tells us that any point has at most one successor. According to [Kracht, 1993], the elementary properties defined by Sahlqvist tense formulae can be described as follows. They are of the form $(\forall x) \Phi$ where $\Phi$ is composed from positive formulae $x \triangleleft y, x=y$ with the help of $\wedge, \vee$ and the so-called two way restricted quantifiers ( $\exists y \triangleright x),(\exists y \triangleleft x),(\forall y \triangleright x),(\forall y \triangleleft x)$ in such a way that in a subformula $x \triangleleft y, x=y$ at least one of $x$ and $y$ is hereditarily universal, which means that it is not inside an existential quantifier. Modal Sahlqvist formulae differ only with respect to the quantifiers $(\exists y \triangleleft x)$ and $(\forall y \triangleleft x)$, which may not be used. Let us call $\phi=(\forall x) \chi$ a primitive formula (modal or tense) if it is of the described form and no universal quantifier is in the scope of an existential quantifier; hence it has the form $(\forall)(\exists) \Psi$ (with the appropriate restricted quantifiers) where $\Psi$ is positive and in an atomic subformula $x \triangleleft y$ or $x=y$ at least one of $x$ and $y$ is hereditarily universal.

Theorem 17 Suppose that $\mathfrak{F}$ is a class of modal or tense Kripke-frames described by some finite set of primitive sentences. Then the modal logic of $\mathfrak{F}$ can be properly displayed.

Proof. It suffices to derive in the calculus of first-order equivalents as described in [Kracht, 1993] that a negative, bounded $\exists \forall$-sentence is equivalent to a sequence $\mathfrak{N} \otimes \mathfrak{P}$ where $\mathfrak{N}$ is negative and free of $\square, \square$ and $\mathfrak{P}$ is positive and free of $\diamond, \diamond . \dashv$ 
This seems surprising, because well-known systems such as $\mathbf{S 5}$, Alt $\mathbf{t}_{1}$ etc. are often axiomatized using $\square(=\square)$. But this need not be so. The axiom of transitivity can be rendered as $\diamond \diamond p \rightarrow \diamond p$, the axiom of reflexivity as $p \rightarrow \diamond p$, and the axiom of symmetry as $p \wedge \diamond q \rightarrow \diamond(q \wedge \diamond p)$. The quasi-functionality can be axiomatized by $\diamond p \wedge \diamond q \rightarrow \diamond(p \wedge q)$ rather than $\diamond p \rightarrow \square p$.

Corollary 18 All elementary subframe logics can be properly displayed.

Proof. If $\Lambda$ is elementary, say, described by $\phi$, then $\phi$ is a restricted universal sentence. Moreover, by a result in [Kracht, 1993] if $\phi$ is universal and it's class is closed under p-morphisms, then $\phi$ is equivalent to a universal and positive sentence. ${ }^{1}$ Thus elementary subframe logics fall under the class just mentioned in the theorem above. $\dashv$

Corollary 19 All r-persistent subframe logics can be properly displayed.

Proof. R-persistent logics are elementary, due to a result by [Fine, 1975]. $\dashv$

\section{Nice Rules and Modal Displayability}

This section discusses the possibility of writing nice rules for modal axioms. Two things will be shown; first, that the extra strength added by the fact that we have the tense dual of the modal operator allows to state rules quite concisely in some cases. And second, that the extra strength does not allow to axiomatize more logics. We will demonstrate the first point with a particular example. Suppose, we are interested in a display rule for $\mathbf{. 3}$.

$$
.3: \diamond p \wedge \diamond q . \rightarrow . \diamond(p \wedge \diamond q) \vee \diamond(q \wedge \diamond p) \vee \diamond(p \wedge q)
$$

This axiom is already in rather perspicuous form, it is primitive and we can translate it directly into a display rule.

$$
\frac{* \bullet *(X \circ * \bullet * Y) \vdash Z \quad * \bullet *(X \circ Y) \vdash Z \quad * \bullet *(Y \circ * \bullet * X) \vdash Z}{* \bullet * X \circ * \bullet * Y \vdash Z}
$$

Semantically, it has the following content.

$$
(\forall w)(\forall x \triangleright w)(\forall y \triangleright w)(x=y \vee y \triangleleft x \vee x \triangleleft y)
$$

\footnotetext{
${ }^{1}$ The proof there is actually highly incomplete, as I recently found out. A full proof can be found in [Kracht, 1999].
} 


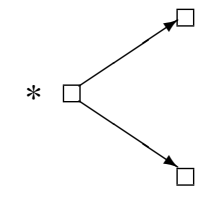

If we are sitting at the root of the tree (marked by a star), thus seeing two points, then these points are either identical or one of them sees the other.

In standard modal logic, one can do no better. However, although it is modal logic we are doing, the display calculus uses a Gentzen toggle and so we have implicitly the power of tense logic in the display calculus. The Gentzen toggle is there anyway, so we can use the extra power it gives us by rewriting the axiom $\mathbf{. 3}$ according to the possibilities of tense logic. Notice, namely, that in tense logic we can generate the tree in the picture from any point we wish because we can look both ways. Consequently, we can shift the reference point of the axiom from the root of the tree to one of the branches.

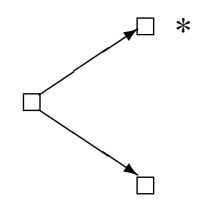

Read from there, the semantic characterization is as follows

$$
(\forall w)(\forall x \triangleleft w)(\forall y \triangleright x)(y=w \vee y \triangleleft w \vee y \triangleright w)
$$

Put into a tense formula it looks like this

$$
\diamond \diamond p . \rightarrow . p \vee \diamond p \vee \diamond p
$$

This axiom uses far less symbols and only one variable. In modal logic, there is no way to axiomatize $\mathbf{S 4 . 3}$ over $\mathbf{S 4}$ with the help of just one variable, even though there are different ways of writing $\mathbf{. 3}$ (but effectively only one primitive way). Hence we can axiomatize . $\mathbf{3}$ in DLM with the following rule

$$
\frac{X \vdash Y \quad X \vdash Y * \bullet * X \vdash Y}{\bullet * \bullet * X \vdash Y}
$$

Now for the second claim that we cannot axiomatize more logics. The Proper Display Theorem I states that a tense logic is properly displayable iff it can be axiomatized by a set of primitive formulae. This can be strengthened to modal logics 
using some model theoretic techniques. Let us begin by introducing generalized restricted quantifiers. We put

$$
\begin{aligned}
& \left(\forall y \triangleright_{0} x\right) \phi(x, y)=\phi(x, x) \\
& \left(\exists y \triangleright_{0} x\right) \phi(x, y)=\phi(x, x) \\
& \left(\forall y \triangleright_{n+1} x\right) \phi(x, y)=\phi(x, x) \wedge(\forall z \triangleright x)\left(\forall y \triangleright_{n} z\right) \phi(x, y) \\
& \left(\exists y \triangleright_{n+1} x\right) \phi(x, y)=\phi(x, x) \vee(\exists z \triangleright x)\left(\exists y \triangleright_{n} z\right) \phi(x, y)
\end{aligned}
$$

These quantifiers quantify over sets of points which can be reached within a fixed number of steps. Recall that a modal formula is called restricted if it is built from atomic formulae with the help of the quantifiers $(\exists y \triangleright x)$, and $(\forall y \triangleright x)$. It is equivalent to require that the generalized restricted quantifiers be used. Our aim is to show that if $\alpha$ is primitive and characterizes a modal class of frames then $\alpha$ is equivalent to a primitive modal formula. The problem here is to get rid of the quantifiers $(\forall y \triangleleft x)$ and $(\exists y \triangleleft x)$. In order to do this, we take a detour. Clearly, $\alpha$ can be written in the form $(\forall \bar{x})(\exists \bar{y}) \phi(\bar{x}, \bar{y})$, with $\phi(\bar{x}, \bar{y})$ quantifier free, using unrestricted quantifiers. To simplify the notation, we use a single variable $x$ instead of $\bar{x}$ and likewise a single variable $y$ for $\bar{y}$. Define now the formulae $\delta_{k}(x)=\left(\exists y \triangleright_{k} x\right) \phi(x, y)$. Then

$$
\delta_{0}(x) \vdash \delta_{1}(x) \vdash \delta_{2}(x) \vdash \ldots \vdash(\exists y) \phi(x, y)
$$

It is therefore enough if we show that there is a $k$ such that $(\exists y) \phi(x, y) \vdash \delta_{k}(x)$. Suppose that this is not so. Then the following set is consistent.

$$
\begin{aligned}
X= & \{(\forall x)(\exists y) \phi(x, y),(\exists y) \phi(u, y), \\
& \left(\forall y \triangleright_{0} u\right) \neg \phi(u, y), \\
& \left(\forall y \triangleright_{1} u\right) \neg \phi(u, y), \\
& \left.\left(\forall y \triangleright_{2} u\right) \neg \phi(u, y), \ldots\right\}
\end{aligned}
$$

Thus there is a model $\mathfrak{M}$ for $X$. Let $\mathfrak{N}$ be the submodel generated by $u$. This model consists of all points which can be reached from $u$ in a finite number of steps. Since $\alpha$ is a modal formula, it is preserved under taking generated submodels and we find that $\mathfrak{N} \vDash \alpha$, by which also $\mathfrak{N} \vDash(\exists y) \phi(u, y)$. Hence for some $w \in \mathfrak{N}, \mathfrak{N} \vDash \phi(u, w)$. Since $\phi$ is constant, it is reflected under generated subframes. So $\mathfrak{M} \vDash \phi(u, w)$ as well and thus $\mathfrak{M}=\left(\exists y \triangleright_{k} u\right) \phi(u, y)$ for some $k$, since $w$ can be reached from $u$ in a finite number of steps, according to the definition of the submodel. This, however, contradicts our assumption on $\mathfrak{M}$. We conclude that $X$ is inconsistent. Hence, we can write $\alpha$ in the form $(\forall x)\left(\exists y \triangleright_{k} x\right) \phi(x, y)$ with $\phi(x, y)$ quantifier-free.

The next problem are the universal quantifiers. Assume that $\alpha=(\forall x)(\forall y)(\exists z) \phi(x, y, z)$. We have seen that we can strengthen the existential quantifier to a restricted one, 
keeping the matrix $\phi$ constant. Now we consider $\neg \alpha$. This sentence is preserved under generated subframes as well. It is of the form $(\exists x)(\exists y) \psi(x, y)$, where $\psi(x, y)$ is restricted. We can perform a similar argument as above, using $\psi(x, y)$ instead of $\phi(x, y, z)$. Notice, namely, that in the proof we have needed only that it is reflected under generated submodels. Thus we can effectively strengthen our formula to $(\forall x)\left(\forall y \triangleright_{k} x\right)\left(\exists z \triangleright_{\ell} x\right) \phi(x, y, z)$. The last task is to remove the negative atomic subformulae. This can be done. According to Theorem 31 of [Kracht, 1993] since $\alpha$ is closed under p-morphic images it is equivalent to a formula $(\forall x)\left(\exists y \triangleright_{k} x\right)\left(\forall z \triangleright_{\ell} x\right) \psi(x, y, z)$ where $\psi(x, y, z)$ results from $\phi(x, y, z)$ by replacing all negative subformulae by either false or true.

Theorem 20 (Proper Display II) An axiomatic extension of Hilbert-style modal logic can be properly displayed (by structural rules over DLM) iff it is axiomatizable by a set of primitive modal axioms.

\section{Polymodal Logics and the Decidability Question}

The most remarkable aspect of the display calculus is that it generalizes easily to logics with several modal operators. Just imagine we have instead of one modal operator (and its tense dual) a finite list $\square_{i}$ of modal operators (possibly together with their tense duals $\diamond_{i}$ ). Then we proceed by redefining Struc, positing a Gentzen toggle $\bullet_{i}$ for each pair $\square_{i}, \diamond_{i}$ and writing down the rules for introducing the operators for each operator independently. The conditions $(C 1)-(C 8)$ are immediately satisfied. Notice that the condition $(C 8)$ is modular in the sense that only the calculi restricted to the individual operators have to be checked for $(C 8)$. If they satisfy it, the overall calculus does so, too. We then have the subformula property and cutelimination. Define primitive of an elementary condition as follows. A first-order $n$-modal sentence is primitive if it is if the form $(\forall x) \chi$ where $\chi$ is produced from atomic formulae $x=y$ with the help of $\wedge, \vee$ and two way restricted quantifiers $\left(\forall x \triangleright_{i} y\right)\left(\forall x \triangleleft_{i} y\right),\left(\exists x \triangleright_{i} y\right)$ and $\left(\exists x \triangleleft_{i} y\right)$ with the extra conditions that no universal quantifier is in the scope of an existential quantifier and in each atomic subformula $x=y$ at least one of $x$ and $y$ is inherently universal.

Theorem 21 Let $\mathfrak{F}$ be a class of Kripke polyframes defined by a finite set of primitive sentences. Then the logic of $\mathfrak{F}$ can be properly displayed. $\dashv$

If such a logic can be properly displayed we can use axioms of the form $A \rightarrow B$ where both $A$ and $B$ are made from variables with the help of $\mathrm{T}, \wedge, \vee, \diamond_{i},\left(\diamond_{i}\right)$ such 
that $A$ contains a variable only once. Again, we call these axioms primitive. All theorems of the preceding section hold in their canonical extension to polymodal logics.

We will use this fact to derive a rather negative result concerning the decidability of display logics. One might think that cut-elimination and the subformula property are enough to have decidability - but the subformula property is too weak to guarantee that we can give a priori bounds on the lengths of sequents occurring in a minimal proof of a given sequent. One might think, then, that it is just a question of being clever enough. However, the opposite is true. We will simply prove here the following theorem.

Theorem 22 It is undecidable whether or not a display calculus is decidable.

The proof involves the simulation of a Thue-process. We produce here a counterexample based on a result found together with C. Grefe. Proofs and exact details can be found in [Kracht, 1995]. ${ }^{2}$ Consider a bimodal logic with operators $\boxplus$ and $\otimes$. We assume that both operators satisfy the axiom $\mathbf{A l t}_{1}$, so that the relation on the Kripke frames satisfies the condition that each point has at most one successor in each relation. Suppose further that both satisfy the axiom $\mathbf{D}$, so that in fact each point has exactly one successor. We will show how to code Thue-processes into such frames as extensions of the $\operatorname{logic} \mathbf{A l t}_{\mathbf{1}} \mathbf{. D} \otimes \mathbf{A} \mathbf{I t}_{\mathbf{1}}$.D. It will turn out that the logics are undecidable if the corresponding Thue-process is and so we have plenty of undecidable finitely axiomatizable logics. So, let $\mathfrak{I}=\left\{u_{i} \sim v_{i} \mid i \in n\right\}$ be a set of equations over strings in the alphabet $\{a, b\}$. Recall that $\mathfrak{I}$ specifies a relation $\sim$ between words as follows. $w \sim_{0} z$ if $w=z ; w \sim_{n+1} z$ iff there is a $y$ such that $w \sim_{n} y$ and $y=\bar{y} u_{i} \overline{\bar{y}}, z=\bar{y} v_{i} \overline{\bar{y}}$ for some $i \leq n$ or $y=\bar{y} v_{i} \overline{\bar{y}}, z=\bar{y} u_{i} \overline{\bar{y}}$. Then $\sim=\bigcup_{k} \sim{ }_{k}$. Such equations can be mimicked by modal axioms. Define first a translation of strings into formulae.

$$
\begin{gathered}
a^{t}=\boxplus, b^{t}=\otimes,(v w)^{t}=v^{t} w^{t} \\
\left(u_{i}=v_{i}\right)^{t}=u_{i}^{t} p \leftrightarrow v_{i}^{t} p
\end{gathered}
$$

For example, $a a b \sim b a$ gets translated into $\boxplus \boxplus \otimes p \leftrightarrow \otimes \boxplus p$. Notice that the axioms are all equivalent to primitive formulae. It can now be shown that $w \sim z$ iff $w^{t} p \leftrightarrow z^{t} p$ is derivable in the logic K.Alt $\mathbf{1} . \mathbf{D} \otimes \mathbf{K} . \mathbf{A l t} \mathbf{1} . \mathbf{D}(\mathfrak{I})$. If $\mathfrak{I}$ is undecidable the corresponding logic is undecidable as well. Moreover, if $\mathfrak{I}$ is decidable, so is the corresponding logic. Hence, for the logics simulating Thue-processes the

\footnotetext{
${ }^{2}$ The relevant results have actually been known to Alexander Muchnik in 1974, as I have been told by Valentin Shehtman. He has not published his findings, though.
} 
question of decidability is undecidable. Since they are all properly displayable logics, Theorem 22 is proved.

Remark. The proof of the theorem requires at least two modal operators. It is not known so far whether for monomodal logics the theorem holds as well.

\section{Speeding up Proofs}

Despite its theoretic advantages, display logic is a rather clumsy tool in actual computation. Anyone having done or tried a few proofs in display logic will see this. We will therefore develop a calculus of compressed proofs that allows to speed up a display proof considerably. The way this problem is attacked is by noting that the rules of the calculus allow to compress the structures that appear during a proof into some smaller structures whose size is bounded a priori from the size of $X$ and $Y$. Recall therefore the definition of $\approx$. The following holds in the classical calculus.

$$
\begin{array}{ll}
X \circ \mathbf{I} & \approx X \\
*(X \circ Y) & \approx * X \circ * Y \\
* * X & \approx X \\
X \circ X & \approx X \\
X \circ Y & \approx Y \circ X \\
X \circ(Y \circ Z) & \approx(X \circ Y) \circ Z
\end{array}
$$

These equivalences are not difficult to show. There might be more valid equations; however, it is enough if the ones given hold and so we fix $\approx$ to be the congruence defined by these particular equations. Especially useful is the normal form theorem.

Definition 23 A structure term is in normal form of rank 0 if it is of the form

$$
P_{1} \circ P_{2} \circ \ldots \circ P_{k} \circ * Q_{1} \circ * Q_{2} \circ \ldots \circ * Q_{l}
$$

where the $P_{i}, Q_{j}$ are formulae; it is called reduced if all $P_{i}$ are different and all $Q_{j}$ are different. A structure term is called in normal form of rank $n+1$ if it is of the form

$$
V \circ \bullet X_{1} \circ \bullet X_{2} \circ \ldots \bullet \circ X_{m} \circ * \bullet Y_{1} \circ * \bullet Y_{2} \circ \ldots \circ * \bullet Y_{n}
$$

where all $V, X_{i}, Y_{j}$ are in normal form of rank $\leq n$. It is called reduced if all $X_{i}$ are different and all $Y_{j}$ are different as well, and if $V$ is reduced. 
Theorem 24 (Normal Forms) Every term $X \in \operatorname{Struc}(\mathcal{L})$ can be brought effectively into normal form $Y \in \operatorname{Struc}(\mathcal{L})$. Moreover, it can be brought effectively into reduced normal form. $\dashv$

Let us denote by $\operatorname{Comp}(\mathcal{L})$ the algebra $\operatorname{Struc}(\mathcal{L}) / \approx$. We will call it the algebra of compressed $\mathcal{L}$-structures. However, instead of working with compressed structures one can also work with reduced normal forms also called reduced structures. Notice that normal forms are not unique, but if $X, Y$ are equivalent normal forms then they contain the same number of symbols, that is, they are of equal length. A reduced structure contains no stacked $*$ unless separated by a $\bullet$, and $*$ is obligatorily distributed over $\circ$. No double occurrences of a structure $X$ are within the same nesting of $\bullet$.

Define now the layer of a structure as follows.

$$
\begin{array}{ll}
\lambda(P) & =0 \\
\lambda(\mathbf{I}) & =0 \\
\lambda(* X) & =\lambda(X) \\
\lambda(X \circ Y) & =\max \{\lambda(X), \lambda(Y)\} \\
\lambda(\bullet X) & =1+\lambda(X)
\end{array}
$$

We can effectively count the number of reduced structures up to a given layer. The bounding number is computed recursively as follows.

$$
\begin{array}{ll}
n(0, \gamma) & =2^{2 \gamma} \\
n(\lambda+1, \gamma) & =n(0, \gamma) \cdot 2^{2 n(\lambda, \gamma)}
\end{array}
$$

Proposition $25 \operatorname{Comp}(G)$ contains exactly $n(\lambda, \sharp G)$ elements of layer $\leq \lambda$.

Proof. It is enough to count the number of nonequivalent reduced normal forms. At layer 0 , we have the form

$$
P_{1} \circ P_{2} \circ \ldots \circ P_{k} \circ * Q_{1} \circ * Q_{2} \circ \ldots \circ * Q_{l}
$$

Two such forms are equivalent iff the sets of unstarred formulae coincide and the sets of starred formulae coincide. There are $2^{\gamma}$ sets of formulae, where $\gamma=\sharp G$ since the generators are assumed different. Thus at layer 0 we have exactly $2^{\gamma} \times 2^{\gamma}=$ $2^{2 \sharp G}$ elements. Suppose then that the elements of layer $\lambda$ are counted by $n(\lambda, \sharp G)$. At layer $\lambda+1$, every element is of the form

$$
X \circ \bullet Y_{1} \circ \bullet Y_{2} \ldots \circ \bullet Y_{m} \circ * \bullet Z_{1} \circ * \bullet Z_{2} \circ \ldots \circ * \bullet Z_{n}
$$


where $X$, the $Y_{i}$ and the $Z_{j}$ are of layer $\lambda$. The $Y_{i}$ are all distinct as well as the $Z_{j}$, though the $X$ need not be distinct from the $Y_{i}$ and the $Y_{i}$ need not be distinct from the $Z_{j}$. Counting the number of such elements gives $n(0, \sharp G) \cdot 2^{2 n(\lambda, \sharp G)}$.

Let us define the compressed calculus to be the display-calculus read as a calculus over Comp rather than Struc. A proof $\Pi$ in the display calculus is translated into a compressed proof $\Pi^{c}$ line-by-line, by translating $X \vdash Y$ to $X / \approx \vdash Y / \approx$. A compressed proof can be uncompressed by translating a sequent $X / \approx \vdash Y / \approx$ into a sequent $X^{\prime} \vdash Y^{\prime}$ where $X^{\prime} \approx X$ and $Y^{\prime} \approx Y$ and then adding some more proof steps. Although the representative can be chosen at random, there is always a way to complete this proof - by definition of $\approx$. We can, however, discern among these choices of uncompressions some good ones that make use only of reduced formulae. So let $u: \operatorname{Comp}(\mathcal{L}) \rightarrow \operatorname{Struc}(\mathcal{L})$ a map with $u(X / \approx) \approx X$, so $u$ is picking representatives from each class. $u$ is called special if $u(X)$ is always reduced. From now on $u$ is assumed to be always special. Let $\left(\Pi^{c}\right)^{u}$ be the line-by-line translation of $\Pi^{c}$ by $u$. As observed several times, $\left(\Pi^{c}\right)^{u}$ is not necessarily a proof in the strict sense, but there is a way to complete the uncompressed version. Namely if

$$
\rho \frac{X_{1} \vdash Y_{1} \ldots X_{n} \vdash Y_{n}}{V \vdash W}
$$

is a line in the uncompressed proof, then it is of the form $\rho=\sigma^{c u}$ where $\sigma$ is an instance of a display-rule. So $\sigma$ is of the form

$$
\sigma \frac{X_{1}^{\prime} \vdash Y_{1}^{\prime} \ldots X_{n}^{\prime} \vdash Y_{n}^{\prime}}{V^{\prime}+W^{\prime}}
$$

where $X_{i} \approx X_{i}^{\prime}, Y_{j} \approx Y_{j}^{\prime}, V \approx V^{\prime}$ and $W \approx W^{\prime}$. We can then first move from $X_{i} \vdash Y_{i}$ to $X_{i}^{\prime} \vdash Y_{i}^{\prime}$ then conclude $V^{\prime} \vdash W^{\prime}$ and the go back to $V \vdash W$.

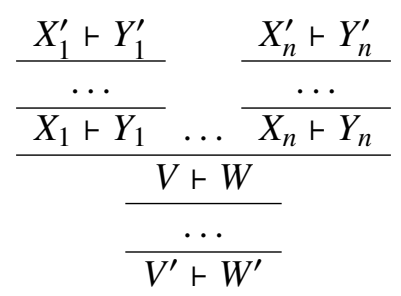

But this proof is at least as complicated as $\Pi$ itself, so this does not amount to a reduction in any sense. The most obvious waste that is produced this way is by completing steps that have been trivialized by this forth-and-back translation. Namely, the rules $(\mathrm{A}),(\mathrm{P}),(\mathrm{C})$ are now completely empty. For example, $(X \circ X)^{c u}=$ 
$X^{c u}$, so if $\rho$ is an instance of $(C)$ then $\rho^{c u}=i d$, the identity transition. Evidently, we can get away by just forgetting about this rule altogether.

But there is a more economical way to complete the proof which goes as follows. Take, for example, the rule $(\vdash \wedge)$.

$$
\frac{X \vdash P \quad X \vdash Q}{X \circ X \vdash P \wedge Q}
$$

Let there be an instance of this rule and let us compress and uncompress this rule. Then we get

$$
\frac{X^{c u} \vdash P \quad X^{c u}+Q}{X^{c u}+P \wedge Q}
$$

There is no need to return to $X \vdash P$ from $X^{c u} \vdash P$ or to $X \vdash Q$ from $X^{c u} \vdash Q$. The rule can be applied directly to the new premisses but it yields the conclusion $X^{c u} \circ X^{c u} \vdash P \wedge Q$ rather than $X^{c u} \vdash P \wedge Q$. However, a single application of $(C l)$ brings this sequent into reduced form. In general, the antecedents are of the form

$$
f\left(X_{1}, \ldots, X_{k}\right) \vdash g\left(X_{1}, \ldots, X_{k}\right)
$$

where the $X_{i}$ need not be different, but occur at most once in $f$ and $g$. Let us write $f(\vec{X})$ instead of $f\left(X_{1}, \ldots, X_{k}\right)$, and $f\left(\vec{X}^{c u}\right)$ for $f\left(X_{1}^{c u}, \ldots, X_{k}^{c u}\right)$. We know that

$$
f(\vec{X})^{c u} \approx f\left(\vec{X}^{c u}\right)
$$

as well as

$$
g(\vec{X})^{c u} \approx g\left(\vec{X}^{c u}\right)
$$

by the fact that $\approx$ is a congruence. Moreover, $f(\vec{X})$ is structurally similar to $f\left(\vec{X}^{c u}\right)$ and $g(\vec{X})$ is similar to $g\left(\vec{X}^{c u}\right)$. Thus the rule in question can be applied to the premisses

$$
f\left(\vec{X}^{c u}\right) \vdash g\left(\vec{X}^{c u}\right)
$$

Thus if the following line occurred in $\Pi$

$$
\frac{f_{1}(\vec{X}) \vdash g_{1}(\vec{X}) \ldots f_{n}(\vec{X}) \vdash g_{n}(\vec{X})}{r(\vec{X}) \vdash s(\vec{X})}
$$


then we will replace it by the following proof:

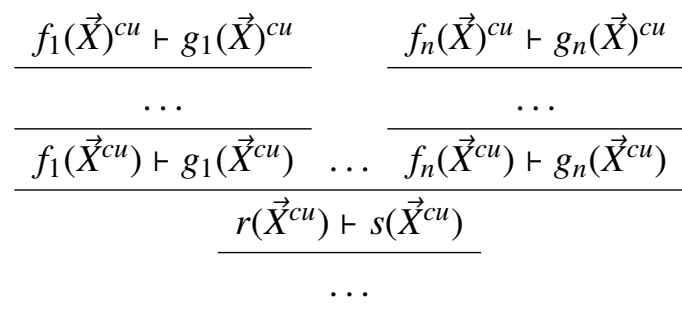

$$
\begin{aligned}
& r(\vec{X}) \vdash s(\vec{X})
\end{aligned}
$$

The starting sequents contain reduced structures and immediately before applying the rule we have structures built from at most $k$ occurrences of reduced structures. So it has no more symbols than a term of $k$ occurrences of reduced structures. This $k$ varies from rule to rule. But we can fix $\widehat{\kappa}$ as the maximum of all $k$ in our calculus. Moreover, we can let $\widehat{\kappa}$ be a bit smaller than that, namely the maximum of occurences of variables in an antecedent or succedent of a rule. Furthermore, rules that are emptied by compression can be left out of consideration. At present, $\widehat{\kappa}=2$. (For (A), where $\widehat{\kappa}=3$, the forth-and-back translation makes the rule void, so we do not have to care about it.)

It should be clear that the number of steps filling the dots in the proof is bounded a priori; one can namely give exact bounds as to how many steps are needed to reduce a structure, and similarly how many are needed to mediate between the sequent $X \vdash Z$ and $Y \vdash Z$ when $X \approx Y$. In order to supply a rigorous argument here we give bounds on the size of intermediate sequents that need occur when passing from $X \vdash Z$ to $Y \vdash Z$. First, let $W$ be reduced and $W \approx X$. Then it is enough to bound the size of sequents for a transformation $X \vdash Z \leadsto W \vdash Z$. The crux is that one cannot simply count the symbols in $X \vdash Z$ and hope that one never needs to use sequents that are longer. Namely, intermediate calculations may involve displaying - and displaying usually means an increase in the length of the structure. Define therefore first the symbol count $s c(X)(s c(X \vdash Y))$ to be the function counting every occurring symbol except brackets (and $\vdash$ ). Then let $\sharp$ be an $n$-ary function and $b_{j}$ it's left or right dual for the $j^{\text {th }}$ position. Define the display factor $\nabla$ as follows.

$$
\nabla:=\max \left\{\frac{s c\left(b_{j}\left(X_{1}, \ldots, X_{n}\right)\right)}{\operatorname{sc}\left(\sharp\left(X_{1}, \ldots, X_{n}\right)\right)} \mid \sharp \text { an } n \text {-ary function symbol and } j \leq n\right\}
$$

By induction it is proved that

$$
\nabla=\sup \left\{\frac{\operatorname{sc}\left(X \vdash f^{\delta}(W)\right)}{s c(f(X) \vdash W)} \mid f \text { a unary structure polynomial }\right\}
$$


Notice that $*$ is included in the definition of $\nabla$ if $*$ counts as it's own dual. The factor $\nabla$ then gives an upper bound for the cost of displaying material at the same side. It is, however, also a bound for displaying at the other side. Namely,

$$
\sup \left\{\frac{s c(* X \vdash Y)}{s c(* Y \vdash X)} \mid X, Y \in \operatorname{Struc}\right\}=1
$$

as well as

$$
\sup \left\{\frac{s c(X \vdash Y)}{s c(* * X \vdash Y)} \mid X, Y \in \operatorname{Struc}\right\}=1
$$

Notice that we do not have to consider the quotient $s c(* * X \vdash Y) / \operatorname{sc}(X \vdash Y)$ because we are interested in removing $*$ from a symbol not adding it. This allows to have $\nabla=3 / 2$ for the current calculus rather than $\nabla=2$.

The algorithm for reducing a sequent consists in displaying structure parts for which a reduction can be applied, applying reduction rules and then undoing the display. Thus, while the reduction parts will actually not increase the length of the sequents it is the display strategy that can increase the length of the structure by a factor $\nabla$. But when we undisplay, the resulting sequent will not be longer than the one we started off with. Thus, the only price to be payed is an increase in the length of occurring structures by the factor $\nabla$.

\section{References}

[Belnap, 1982] Nuel D. Jr. Belnap. Display logic. Journal of Philosophical Logic, 11:375 - 417, 1982.

[Benthem, 1984] Johan van Benthem. Correspondence theory. In Dov M. Gabbay and Franz Guenthner, editors, Handbook of Philosophical Logic, volume 2, pages 167 - 247. Reidel, 1984.

[Fine, 1975] Kit Fine. Some connections between elementary and modal logic. In Stig Kanger, editor, Proceedings of the Third Scandinavian Logic Symposium, pages $15-31$. North-Holland, 1975.

[Kracht, 1991] Marcus Kracht. Internal Definability and Completeness in Modal Logic. PhD thesis, FU Berlin, 1991.

[Kracht, 1993] Marcus Kracht. How completeness and correspondence theory got married. In Maarten de Rijke, editor, Diamonds and Defaults, Synthese, pages 175 - 214. Kluwer, 1993. 
[Kracht, 1995] Marcus Kracht. Highway to the danger zone. Journal of Logic and Computation, 5:93 - 109, 1995.

[Kracht, 1999] Marcus Kracht. Tools and Techniques in Modal Logic. Number 142 in Studies in Logic. Elsevier, Amsterdam, 1999.

[Sahlqvist, 1975] Hendrik Sahlqvist. First and second order semantics for modal logic. In Stig Kanger, editor, Proceedings of the Third Scandinavian Logic Symposium, pages 15 - 31. North-Holland, 1975.

[Wansing, 1994a] Heinrich Wansing. A full circle theorem for simple tense logic. In Maarten de Rijke, editor, Recent Advances in Modal and Intensional Logic. 1994. to appear.

[Wansing, 1994b] Heinrich Wansing. Sequent calculi for normal modal propositional logics. Journal of Logic and Computation, 4, 1994. to appear.

[Wansing, 1994c] Heinrich Wansing. Strong cut-elimination in Display Logic. 1994. 